Check for updates

For numbered affiliations see end of the article

Correspondence to: I Izawa

izawa.junichi.87r@st.kyoto-u.ac.jp (ORCID 0000-0003-1920-8218)

Additional material is published online only. To view please visit the journal online.

Cite this as: $B M / 2019 ; 364: 1430$ http://dx.doi.org/10.1136/bmj.1430

Accepted: 21 January 2019

\title{
Pre-hospital advanced airway management for adults with out-of-hospital cardiac arrest: nationwide cohort study
}

\author{
Junichi Izawa, ${ }^{1,2}$ Sho Komukai, ${ }^{3}$ Koichiro Gibo, ${ }^{4}$ Masashi Okubo, ${ }^{5}$ Kosuke Kiyohara, ${ }^{6}$ \\ Chika Nishiyama, ${ }^{7}$ Takeyuki Kiguchi, ${ }^{8}$ Tasuku Matsuyama, ${ }^{9}$ Takashi Kawamura, ${ }^{8}$ Taku Iwami, ${ }^{8}$ \\ Clifton W Callaway, ${ }^{5}$ Tetsuhisa Kitamura ${ }^{10}$
}

\section{ABSTRACT}

OBJECTIVE

To determine survival associated with advanced airway management (AAM) compared with no AAM for adults with out-of-hospital cardiac arrest.

\section{DESIGN}

Cohort study between January 2014 and December 2016.

\section{SETTING}

Nationwide, population based registry in Japan (AllJapan Utstein Registry).

\section{PARTICIPANTS \\ Consecutive adult patients with out-of-hospital cardiac arrest, separated into two sub-cohorts by their first documented electrocardiographic rhythm: shockable (ventricular fibrillation or pulseless ventricular tachycardia) and non-shockable (pulseless electrical activity or asystole). Patients who received AAM during cardiopulmonary resuscitation were sequentially matched with patients at risk of AAM within the same minute on the basis of time dependent propensity scores.}

MAIN OUTCOME MEASURES

Survival at one month or at hospital discharge within one month.

RESULTS

Of the 310620 patients eligible, 8459 (41.2\%) of 20516 in the shockable cohort and 121890 (42.0\%) of 290104 in the non-shockable cohort received AAM during cardiopulmonary resuscitation. After time dependent propensity score sequential matching, 16114 patients in the shockable cohort and 236042

\section{WHAT IS ALREADY KNOWN ON THIS TOPIC}

Many previous observational studies reported unfavourable associations between pre-hospital advanced airway management and patients' outcomes after out-of-hospital cardiac arrest

A recent randomised controlled non-inferiority trial failed to show non-inferiority of bag-valve-mask ventilation versus endotracheal intubation, with more complications in the former group

\section{WHAT THIS STUDY ADDS}

In this study of 310620 adults with out-of-hospital cardiac arrest, results differed according to each patient's first documented electrocardiographic rhythm

Advanced airway management was not associated with survival in the shockable cohort but was associated with better survival in the non-shockable cohort, although the absolute effect was very small

These findings suggest that different airway management strategies should be emphasised on the basis of the initial electrocardiographic rhythm: shockable or non-shockable in the non-shockable cohort were matched at the same minute. In the shockable cohort, survival did not differ between patients with AAM and those with no AAM: $1546 / 8057$ (19.2\%) versus 1500/8057 (18.6\%) (adjusted risk ratio 1.00, 95\% confidence interval 0.93 to 1.07). In the non-shockable cohort, patients with AAM had better survival than those with no AAM: $2696 / 118021$ (2.3\%) versus 2127/118 021 (1.8\%) (adjusted risk ratio $1.27,1.20$ to 1.35 ).

\section{CONCLUSIONS}

In the time dependent propensity score sequential matching for out-of-hospital cardiac arrest in adults, AAM was not associated with survival among patients with shockable rhythm, whereas AAM was associated with better survival among patients with nonshockable rhythm.

\section{Introduction}

Basic life support, which consists of early cardiopulmonary resuscitation and defibrillation with automated external defibrillators, ${ }^{1}$ improves outcomes in patient with out-of-hospital cardiac arrest. ${ }^{2} 3$ Provision of oxygen and ventilation of the lungs via bag-valve-mask ventilation, supraglottic airway placement, or endotracheal intubation are important resuscitation skills for emergency medical services (EMS) personnel. ${ }^{4}$ Supraglottic airway placement and endotracheal intubation constitute advanced airway management (AAM), which secures better protection for the airway than bag-valve-mask ventilation does. Although AAM is a common practice, the risk-benefit ratio of $\mathrm{AAM}$ during pre-hospital resuscitation is unclear. $^{5-7}$

In observational studies assessing intra-cardiac arrest interventions, "resuscitation time bias" (that is, the fact that patients undergoing longer resuscitation tend to receive more interventions) is a crucial bias. ${ }^{8}$ Because of this bias effect, two observational studies that evaluated the impact of adrenaline (epinephrine) on patients with out-of-hospital cardiac arrest, using the same national database in Japan but different statistical approaches, reported conflicting findings. ${ }^{910}$ One study used traditional propensity score matching, ${ }^{9}$ whereas the other used time dependent propensity score sequential matching, accounting for resuscitation time bias and timing of administration of adrenaline. ${ }^{10}$ A recent, large randomised controlled trial showed better survival in the adrenaline group than the placebo group, ${ }^{11}$ and these findings were closer to the results of the second observational study from Japan. ${ }^{10} 12$ This confirms the importance of overcoming resuscitation time bias in observational studies. ${ }^{12}$ Previous studies 
of in-hospital cardiac arrest have evaluated the effect of intubation while accounting for resuscitation time bias and timing of intubation, ${ }^{13}{ }^{14}$ but no studies among patients with out-of-hospital cardiac arrest have considered the timing of AAM.

In Japan, a nationwide registry includes approximately 368000 adult patients with out-ofhospital cardiac arrest with available data on the timing of pre-hospital AAM between 2014 and 2016. This study investigated whether AAM by EMS personnel increased survival for adult patients with out-ofhospital cardiac arrest, adjusting for time dependent interventions and covariates.

\section{Methods}

Study design and setting

We used the All-Japan Utstein Registry database to do a cohort study with time dependent propensity score sequential matching. The All-Japan Utstein Registry of the Fire and Disaster Management Agency is a prospective, population based, nationwide registry of out-of-hospital cardiac arrest launched in January 2005, which records data according to the international Utstein-style reporting system. ${ }^{15}$ Details of the registry have been described previously. ${ }^{16}$ The registry started collecting time stamp data for successful AAM by outof-hospital cardiac arrest personnel in January 2014. Cardiac arrest was defined as the cessation of cardiac mechanical activity, as confirmed by the absence of signs of circulation. ${ }^{15}$ Each EMS authority submitted anonymised data.

\section{Emergency medical services system in Japan}

The EMS system in Japan was previously described elsewhere. ${ }^{5} 16$ Briefly, pre-hospital life support is provided 24 hours each day by a fire station based EMS system. Among EMS personnel, specially trained emergency care providers known as emergency life saving technicians are authorised to use automated external defibrillators. With support and direction from online medical control, emergency life saving technicians are also authorised to perform advanced life support such as administering adrenaline and placing advanced airway for patients with out-ofhospital cardiac arrest. In Japan, EMS providers are generally not permitted to terminate resuscitation in the field, and all patients on whom resuscitation is attempted are transported to hospitals. Resuscitation is not attempted only under particular conditions (for example, decapitation, incineration, decomposition, rigor mortis, or dependent cyanosis). Although all emergency life saving technicians can use supraglottic airway devices such as laryngeal tubes or laryngeal masks, only specially trained, certified emergency life saving technicians are permitted to perform endotracheal intubation under direction from online medical control. These technicians are allowed to perform endotracheal intubation but only during cardiac arrest (that is, they are not allowed to intubate after return of spontaneous circulation). Treatments for cardiac arrest were based on the Japanese cardiopulmonary resuscitation guidelines, ${ }^{17}$ which are based on the guidelines of the consensus of the International Liaison Committee on Resuscitation. ${ }^{18}$

\section{Study participants}

We screened the data of all consecutive cases of outof-hospital cardiac arrest between January 2014 and December 2016. We included patients according to the following inclusion criteria: age 18 years or over; cardiac arrest before arrival of EMS personnel; cardiac arrest for which EMS personnel attempted cardiopulmonary resuscitation; and cardiac arrest attended by an emergency life saving technician. The exclusion criteria were outliers with regard to age (that is, older than 120 years); unknown first documented electrocardiographic rhythm; missing or contradictory data (negative values) in time dependent variables (including time from cardiopulmonary resuscitation by EMS personnel to AAM at the scene, defibrillation at the scene, administration of adrenaline at the scene, and return of spontaneous circulation at the scene; time from call to cardiopulmonary resuscitation by EMS personnel; and time from cardiopulmonary resuscitation by EMS personnel to arrival at hospital); or time from call to cardiopulmonary resuscitation by EMS personnel 30 minutes or more.

\section{Data collection and quality control}

Data were collected prospectively on resuscitation related factors including age and sex of the patient, type of witness status (by family members, friends, colleagues, passers-by, or others), type of bystander initiated cardiopulmonary resuscitation (chest compressions only without rescue breathing or conventional cardiopulmonary resuscitation with rescue breathing), instruction on cardiopulmonary resuscitation by dispatcher, shock delivery by public access automated external defibrillators, presence of emergency life saving technicians, pre-hospital involvement of physician, and first documented electrocardiographic rhythm (ventricular fibrillation, pulseless ventricular tachycardia, pulseless electrical activity, or asystole), as well as return of spontaneous circulation before arrival at hospital, survival, and functional status at one month after the event. On the basis of the consensus guidelines, ${ }^{15}{ }^{19}$ return of spontaneous circulation was defined according to a clinical assessment that shows signs of life comprising a palpable pulse or generation of a blood pressure. Origin of cardiac arrest (medical or non-medical) was also documented; arrest was presumed to be of medical origin unless it was caused by trauma, drug overdose, drowning, electrocution, or asphyxia, according to the current Utstein-style template. ${ }^{19}$ Prefecture and year, month, day, and time when EMS received the emergency call were documented. The times of the receipt of the emergency call, the initiation of cardiopulmonary resuscitation by EMS personnel, the first defibrillation by EMS personnel, the first administration of adrenaline by EMS personnel, return of spontaneous circulation before arrival at hospital, 
the successful achievement of AAM by EMS personnel, and arrival at hospital were recorded according to the times on the clock used by each emergency medical services system.

All survivors were followed for up to one month after out-of-hospital cardiac arrest by the EMS providers who had provided their emergency care. Functional outcomes were determined by the physician responsible for the care of the patient by a follow-up interview at one month after successful resuscitation, with the use of the Cerebral Performance Category scale as follows: 1 , good cerebral performance; 2 , moderate cerebral disability; 3, severe cerebral disability; 4, coma or vegetative state; and 5 , death or brain death. ${ }^{15}$ When the patient was discharged from the hospital before one month, the score at discharge was recorded but the database did not include the information about when the patients were discharged from the hospital.

EMS personnel filled out the study data form in cooperation with the physician in charge of the patient, and the data were stored in the registry system on the Fire and Disaster Management Agency database server. The data were checked for consistency by the computer system and were confirmed by the Fire and Disaster Management Agency. If the data form was incomplete, the Fire and Disaster Management Agency returned it to the respective fire station where it was then completed.

\section{Outcomes}

The primary outcome was survival at one month or at discharge from hospital within one month. The secondary outcome was favourable functional survival at one month or at hospital discharge within one month. We defined favourable functional survival as a Cerebral Performance Category scale of 1 or 2 .

\section{Time dependent propensity scores and sequential matching}

The main exposure was the successful achievement of AAM by EMS personnel. We separated the patients into two sub-cohorts according to their first documented electrocardiographic rhythm-shockable (ventricular fibrillation or pulseless ventricular tachycardia) or non-shockable (pulseless electrical activity or asystole)-because the current international guidelines propose two different algorithms according to the rhythm, with different suggestions regarding the timing of AAM. ${ }^{2021}$ To adjust for all measured potential confounders, we did time dependent propensity score sequential matching analysis in each original cohort. ${ }^{22}$ To estimate the time dependent propensity scores for receipt of AAM during cardiopulmonary resuscitation, we applied the Fine-Gray model in the presence of competing risk with time dependent covariates. ${ }^{23}$ In the regression model, we dealt with pre-hospital return of spontaneous circulation before AAM as the competing risk. We also dealt with arrival at hospital as censoring in the model. The Cox regression model with or without time dependent covariates was used in previous cardiac arrest studies with time dependent propensity score sequential matching. ${ }^{101314}$ However, we regarded pre-hospital return of spontaneous circulation before AAM as informative censoring in the time dependent propensity score model, because AAM during cardiopulmonary resuscitation never occurs after return of spontaneous circulation except in rare cases involving re-arrest. If we regarded pre-hospital return of spontaneous circulation as non-informative censoring, the propensity scores in patients with return of spontaneous circulation before AAM would be overestimated. ${ }^{24}$ The time dependent covariates were defibrillation by EMS personnel and administration of adrenaline by EMS personnel. The time independent covariates in the propensity score predicting model are shown in table 1 and described in the supplementary methods. We included prefecture categories according to the proportions of patients who received supraglottic airway placement $(<15 \%, 15 \%$ to $<30 \%, 30 \%$ to $<45 \%$, $\geq 45 \%)$ and endotracheal intubation $(<5 \%, 5 \%$ to $<10 \%$, $10 \%$ to $<15 \%, \geq 15 \%$ ) by EMS personnel as prefecture elements as applied in a previous study. ${ }^{10}$ Although we adopted the same variables in the time dependent propensity score model for both the shockable and non-shockable original cohorts, we calculated the time dependent propensity scores separately in each rhythm cohort.

On the basis of the predicted time dependent propensity scores, we separately and sequentially propensity score matched each patient receiving AAM at any given minute (from minute 0 to minute 59) with a patient who was at risk of receiving AAM within the same minute. ${ }^{22}$ Sequential matching attenuates resuscitation time bias. ${ }^{825}$ In sequential matching, at risk patients included those who were still undergoing cardiopulmonary resuscitation at the scene and had not yet received AAM before or within the same minute. In addition, replacement of unexposed patients was possible-that is, matched controls with no AAM in a risk set at each timing were allowed to match again later until they received AAM. At risk patients therefore also included patients who received AAM later, as the matching was not dependent on future events. ${ }^{822} 25$

We sequentially matched patients with AAM to those without AAM who had the nearest propensity score in a one to one fashion. We set the calliper width for nearest neighbour matching at 0.2 standard deviations of the propensity score in the logit scale, as recommended. ${ }^{26} 27$ We calculated standardised differences to evaluate the balance of variables in each predicted propensity score matched cohort. We first regarded standardised differences less than 0.1 as having well matched balance, ${ }^{27}$ but we could not achieve the value for the variable of "defibrillation before matching" in the shockable cohort even with a very narrow calliper width (0.001). When we attempted to achieve better balancing of standardised differences $(<0.1)$ by setting the calliper width much narrower $(<0.001)$, we lost a large number of patients. In the end, we decided to avoid losing these patients by using a tight range of target and chose a value of 0.25 rather than 0.1 of standardised differences, as some 


\begin{tabular}{|c|c|c|c|c|c|c|}
\hline \multirow[b]{2}{*}{ Characteristics } & \multicolumn{3}{|l|}{ Shockable rhythm } & \multicolumn{3}{|l|}{ Non-shockable rhythm } \\
\hline & No AAM $(n=12057)$ & AAM $(n=8459)$ & Standardised difference & No AAM $(n=168214)$ & AAM $(n=121890)$ & Standardised difference \\
\hline \multicolumn{3}{|l|}{ Year: } & \multirow{4}{*}{0.04} & & & \multirow{4}{*}{0.02} \\
\hline 2014 & $3964(32.9)$ & $2923(34.6)$ & & $55652(33.1)$ & $41052(33.7)$ & \\
\hline 2015 & $3962(32.9)$ & $2738(32.4)$ & & $55831(33.2)$ & $40681(33.4)$ & \\
\hline 2016 & $4131(34.3)$ & $2798(33.1)$ & & $56731(33.7)$ & $40157(32.9)$ & \\
\hline \multicolumn{3}{|l|}{ Season: } & \multirow{5}{*}{0.03} & & & \multirow{5}{*}{0.04} \\
\hline Spring & 2899 (24.0) & $2010(23.8)$ & & $41363(24.6)$ & $30246(24.8)$ & \\
\hline Summer & $2713(22.5)$ & $1903(22.5)$ & & $32802(19.5)$ & $22555(18.5)$ & \\
\hline Autumn & $2915(24.2)$ & $1952(23.1)$ & & $38850(23.1)$ & $27476(22.5)$ & \\
\hline Winter & $3530(29.3)$ & $2594(30.7)$ & & $55199(32.8)$ & $41613(34.1)$ & \\
\hline \multicolumn{3}{|l|}{ Day: } & \multirow[t]{3}{*}{0.02} & & & \multirow[t]{3}{*}{0.007} \\
\hline Weekday (Monday-Friday) & $8563(71.0)$ & $5928(70.1)$ & & $119328(70.9)$ & $86065(70.6)$ & \\
\hline Weekend (Saturday-Sunday) & $3494(29.0)$ & $2531(29.9)$ & & $48886(29.1)$ & $35825(29.4)$ & \\
\hline \multicolumn{3}{|l|}{ Time: } & \multirow{3}{*}{0.08} & & & 0.01 \\
\hline Daytime (9:00-16:59) & $5311(44.0)$ & $3393(40.1)$ & & $59700(35.5)$ & $42703(35.0)$ & \\
\hline Night time (17:00-8:59) & $6746(56.0)$ & $5066(59.9)$ & & $108514(64.5)$ & $79187(65.0)$ & \\
\hline $\begin{array}{l}\text { Prefecture preference categories for } \\
\text { performing supraglottic airway placement }\end{array}$ & & & & & & \\
\hline$\langle 15 \%$ & $1476(12.2)$ & $275(3.3)$ & 0.624 & $41448(24.6)$ & $7885(6.5)$ & 0.675 \\
\hline $15 \%$ to $<30 \%$ & $5210(43.2)$ & $2232(26.4)$ & & $57645(34.3)$ & $30660(25.2)$ & \\
\hline $30 \%$ to $<45 \%$ & $1950(16.2)$ & $1313(15.5)$ & & $31710(18.9)$ & $27752(22.8)$ & \\
\hline$\geq 45 \%$ & $3421(28.4)$ & $4639(54.8)$ & & $37411(22.2)$ & $55593(45.6)$ & \\
\hline $\begin{array}{l}\text { Prefecture preference categories for } \\
\text { performing endotracheal intubation: }\end{array}$ & & & & & & \\
\hline$<5 \%$ & $8684(72.0)$ & $5777(68.3)$ & 0.15 & $70824(42.1)$ & $34594(28.4)$ & 0.32 \\
\hline $5 \%$ to $<10 \%$ & $1015(8.4)$ & $575(6.8)$ & & $53488(31.8)$ & $47234(38.8)$ & \\
\hline $10 \%$ to $<15 \%$ & $1117(9.3)$ & $1140(13.5)$ & & $11180(6.6)$ & $6338(5.2)$ & \\
\hline$\geq 15 \%$ & $1241(10.3)$ & 967 (11.4) & & $32722(19.5)$ & $33724(27.7)$ & \\
\hline Median (IQR) age, years & $68(57-78)$ & $69(59-79)$ & 0.07 & $80(69-87)$ & $80(70-87)$ & 0.04 \\
\hline Sex: & & & 0.02 & & & 0.05 \\
\hline Female & $2733(22.7)$ & $1847(21.8)$ & .02 & $77497(46.1)$ & $52957(43.4)$ & \\
\hline Male & $9324(77.3)$ & $6612(78.2)$ & & $90717(53.9)$ & $68933(56.6)$ & \\
\hline Cause of cardiac arrest: & & & 0.02 & & & 0.12 \\
\hline Medical & $11238(93.2)$ & $7924(93.7)$ & & $121506(72.2)$ & $94491(77.5)$ & \\
\hline Non-medical & $819(6.8)$ & $535(6.3)$ & & $46708(27.8)$ & $27399(22.5)$ & \\
\hline Witness status: & & & & & & \\
\hline No witness & $3336(27.7)$ & $2693(31.8)$ & 0.17 & $115063(68.4)$ & $78767(64.6)$ & 0.12 \\
\hline By family & $4866(40.4)$ & $3673(43.4)$ & & $30545(18.2)$ & $27329(22.4)$ & \\
\hline By friend & $720(6.0)$ & $425(5.0)$ & & $1561(0.9)$ & $1413(1.2)$ & \\
\hline By colleague & $903(7.5)$ & $486(5.7)$ & & $1219(0.7)$ & $926(0.8)$ & \\
\hline By passer-by & $713(5.9)$ & $375(4.4)$ & & $2857(1.7)$ & $1393(1.1)$ & \\
\hline By others & $1519(12.6)$ & $807(9.5)$ & & $16969(10.1)$ & $12062(9.9)$ & \\
\hline Type of bystander CPR: & & & & & & \\
\hline No bystander CPR & 5051 (41.9) & $3730(44.1)$ & & $86232(51.3)$ & $56574(46.4)$ & \\
\hline $\begin{array}{l}\text { Chest compression only CPR with } \\
\text { dispatcher instruction }\end{array}$ & $4527(37.5)$ & $3343(39.5)$ & & $56981(33.9)$ & $48035(39.4)$ & \\
\hline $\begin{array}{l}\text { Chest compression only CPR without } \\
\text { dispatcher instruction }\end{array}$ & $1513(12.5)$ & $816(9.6)$ & 0.13 & $15003(8.9)$ & $9666(7.9)$ & 0.13 \\
\hline $\begin{array}{l}\text { Conventional CPR with dispatcher } \\
\text { instruction }\end{array}$ & $545(4.5)$ & $390(4.6)$ & & $6037(3.6)$ & $5272(4.3)$ & \\
\hline $\begin{array}{l}\text { Conventional CPR without dispatcher } \\
\text { instruction }\end{array}$ & $421(3.5)$ & $180(2.1)$ & & $3961(2.4)$ & $2343(1.9)$ & \\
\hline $\begin{array}{l}\text { Use of public access defibrillator by } \\
\text { bystander: }\end{array}$ & & & 0.10 & & & 0.002 \\
\hline Yes & $732(6.1)$ & $335(4.0)$ & & $1408(0.8)$ & $1040(0.9)$ & \\
\hline No & $11325(93.9)$ & $8124(96.0)$ & & $166806(99.2)$ & $120850(99.1)$ & \\
\hline Pre-hospital involvement of physician: & & & 0.03 & & & 0.05 \\
\hline Yes & 709 (5.9) & $442(5.2)$ & & $5384(3.2)$ & $2969(2.4)$ & \\
\hline No & $11348(94.1)$ & $8017(94.8)$ & & $162830(96.8)$ & $118921(97.6)$ & \\
\hline $\begin{array}{l}\text { Median (IQR) time from call to EMS CPR, } \\
\text { minutes }\end{array}$ & $8(7-10)$ & $9(7-11)$ & 0.14 & $9(7-11)$ & $9(7-11)$ & 0.04 \\
\hline Defibrillation by EMS: & & & & & & \\
\hline Yes & $11435(94.8)$ & $8184(96.7)$ & 10 10 & $4767(2.8)$ & $5178(4.2)$ & 808 \\
\hline $\begin{array}{l}\text { Median (IQR) time from EMS CPR to } \\
\text { a }\end{array}$ & & & & & & \\
\hline $\begin{array}{l}\text { defibrillation, minutes } \\
\text { No }\end{array}$ & $\frac{1(1-2)}{622(5.2)}$ & $\frac{1(1-2)}{275(3.3)}$ & & $\begin{array}{l}9(5-15) \\
163447(97.2)\end{array}$ & $\frac{12(6-19)}{116712(95.8)}$ & \\
\hline Administration of adrenaline by EMS: & & & & & & \\
\hline Yes & $2614(21.7)$ & $4023(47.6)$ & 0.57 & $16103(9.6)$ & $35790(29.4)$ & 0.52 \\
\hline Median (IQR) time from EMS CPR to & & & & & & \\
\hline adrenaline administration, minutes & $13(9-17)$ & $13(9-18)$ & & $14(10-18)$ & $14(10-19)$ & \\
\hline No & $9443(78.3)$ & $4436(52.4)$ & & $152111(90.4)$ & $86100(70.6)$ & \\
\hline
\end{tabular}

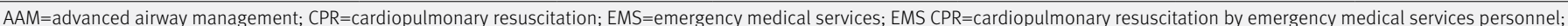
IQR=interquartile range. 
statisticians have suggested, ${ }^{26}$ before doing our final analyses. After sequential matching, we calculated the time from cardiopulmonary resuscitation by EMS personnel to sequential matching.

\section{Statistical analysis}

We did all the analyses separately for each of the two cohorts defined by the first documented electrocardiographic rhythm. We presented data as medians with an interquartile range for continuous variables and as proportions for categorical variables. We used standardised differences for the description of the data in both the original cohorts and the sequentially matched cohorts. We estimated unadjusted and adjusted risk ratios on the primary and secondary outcomes by multivariable regression to evaluate overall outcomes in the original cohorts (see the supplementary methods). Matched controls within a risk set stratum in each minute were independent from each other, whereas some controls in the matched cohort after jointing of all strata could be duplicated and were no longer independent. In the outcome analysis stage after matching, we handled this problem by adjusting for frequency weights that indicate the number of duplicated controls. ${ }^{26}$ Among all risk set strata in each minute, treated patients were paired up with their respective controls, creating within pair correlations. At the outcome analysis stage, therefore, we used generalised estimating equations to estimate the unadjusted and adjusted risk ratios for the primary and secondary outcomes, considering within pair correlation of sequential matching at the same timing. ${ }^{27}$ We adjusted risk ratios for defibrillation before matching, adrenaline administration before matching, and all the time independent covariates in the propensity score predicting model.

To confirm the consistency of the effects between the types of AAM, we stratified the patients into two groups according to each patient's type of AAM (supraglottic airway placement or endotracheal intubation) and calculated adjusted risk ratios with the same multivariable model used in the primary analysis. In this stratified analysis, we picked the pairs of supraglottic airway placement and the pairs of endotracheal intubation out of the sequentially matched cohorts. Therefore, patients were still nested within pairs at the same timing, as we selected the control from within each pair of patients-that is, the sequentially matched pairs in each risk set stratum were not collapsed even after the stratification. As a sensitivity analysis, we also did traditional, non-time dependent propensity score matching analysis as described in the supplementary methods.

We used R (R Foundation for Statistical Computing, version 3.4.3) for all statistical analyses. We did not impute missing data because no information was missing in any key variables after we excluded patients according to the exclusion criteria described above. We calculated all risk ratios with their 95\% confidence intervals by log-binomial regression in generalised estimating equations with the robust variance estimators. All tests were two tailed; we regarded $\mathrm{P}$ values of less than 0.05 as statistically significant. The entire author group outlined and agreed on the statistical analysis plan before we did any analyses, unless stated otherwise. The other details are described in the supplementary methods.

\section{Patient and public involvement}

No patients were involved in setting the research question or the outcome measures, nor were they involved in developing plans for recruitment, design, or implementation of the study. No patients were asked to advise on interpretation or writing up of results. There are no plans to disseminate the results of the research to study participants or the relevant patient community.

\section{Results}

\section{Original cohort and outcomes}

We screened all 372926 cases of out-of-hospital cardiac arrest from the All-Japan Utstein Registry during 2014-16. We divided the 310620 adult patients who met our eligibility criteria into two subcohorts according to each patient's first documented electrocardiographic rhythm; 20516 had shockable rhythm and 290104 had non-shockable rhythm (fig 1). Table 1 shows the characteristics of the patients in the original cohort. Among the patients with shockable rhythm, 8459 (41.2\%) received AAM; among patients with non-shockable rhythm, 121890 (42.0\%) received AAM. Among patients who received AAM, the median time from cardiopulmonary resuscitation by EMS personnel to AAM was 8 (interquartile range 5-12) minutes in the shockable cohort and 8 (5-12) minutes in the non-shockable cohort. AAM was associated with worse survival in both rhythm cohorts, with multivariable regression adjusting for covariates: $1625 / 8459$ (19.2\%) versus 4277/12057 (35.5\%), adjusted risk ratio 0.61 (95\% confidence interval 0.58 to 0.65 ) in the shockable cohort; and $2783 / 121890$ (2.3\%) versus 3939/168214 (2.3\%), adjusted risk ratio 0.82 ( 0.78 to 0.86$)$ in the non-shockable cohort (supplementary table A).

\section{Sequentially matched cohort}

After sequential matching, 16114 patients in the shockable cohort and 236042 in the non-shockable cohort were matched (table 2). All of the variables incorporated into the propensity score model were well balanced in both cohorts except for defibrillation by EMS personnel before matching in the shockable cohort (standardised difference 0.19). In both the shockable and non-shockable cohorts, the largest numbers of patients were matched during five to less than 10 minutes from the time EMS personnel started cardiopulmonary resuscitation: 7040 (43.7\%) in the shockable cohort and $98410(41.7 \%)$ in the non-shockable cohort. Among patients who had not yet received AAM (that is, patients in the "no AAM" group), $2605 / 8057(32.3 \%)$ in the shockable cohort 


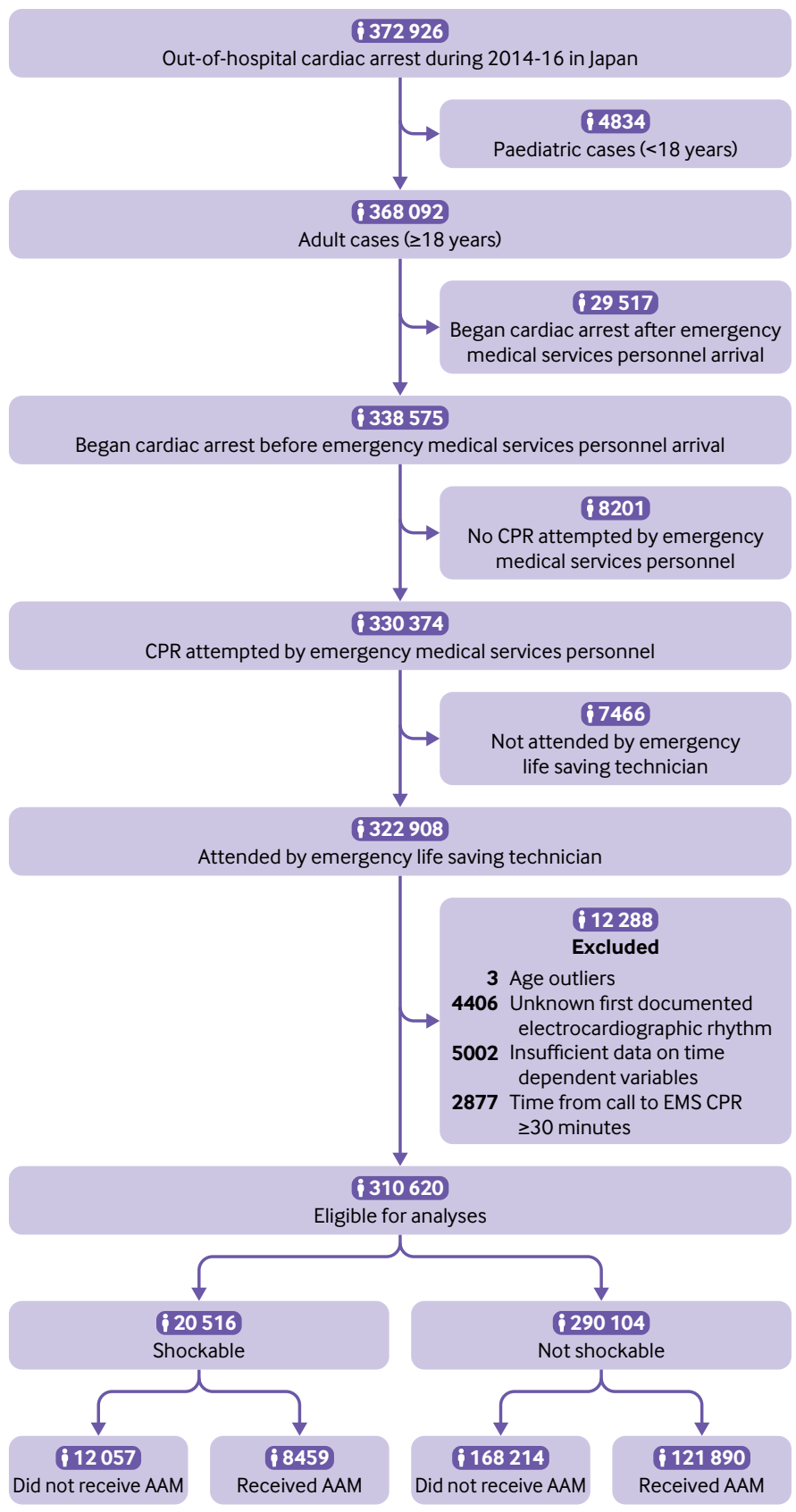

Fig 1 | Flow of out-of-hospital cardiac arrest cases from All-Japan Utstein Project Database between January 2014 and December 2016. AAM=advanced airway management; $C P R=$ cardiopulmonary resuscitation; $E M S=$ emergency medical services; $E M S C P R=$ cardiopulmonary resuscitation by emergency medical services personnel

and $35920 / 118021(30.4 \%)$ in the non-shockable cohort received AAM later.

\section{Outcomes in sequentially matched cohort of} shockable rhythm

In the shockable cohort, survival did not differ between patients who received AAM and those who did not receive AAM (1546/8057 (19.2\%) versus 1500/8057 (18.6\%); adjusted risk ratio $1.00,0.93$ to 1.07 ) (table 3). Patients who received AAM had lower risks of favourable functional survival (776/8057 (9.6\%) versus $865 / 8057$ (10.7\%); adjusted risk ratio 0.87 ,
0.79 to 0.96$)$. When we stratified the matched patients by the type of AAM (supplementary table B), neither supraglottic airway placement nor endotracheal intubation was associated with survival: adjusted risk ratios 1.00 (0.93 to 1.07) for supraglottic airway placement and 0.97 (0.79 to 1.18) for endotracheal intubation (fig 2).

\section{Outcomes in sequentially matched cohort of non- shockable rhythm}

In the non-shockable cohort, patients with AAM had greater risks of survival $(2696 / 118021(2.3 \%)$ versus $2127 / 118021$ (1.8\%); adjusted risk ratio $1.27,1.20$ to 1.35) (table 3). Favourable functional survival did not differ between patients who received AAM and those who did not receive AAM (499/118021 (0.4\%) versus $489 / 118021$ (0.4\%); adjusted risk ratio $1.11,0.97$ to 1.26). When we stratified the matched patients by the type of AAM (supplementary table C), both supraglottic airway placement and endotracheal intubation were associated with survival: adjusted risk ratios were 1.11 (1.03 to 1.19) for supraglottic airway placement and 2.00 (1.74 to 2.30) for endotracheal intubation (fig 2).

\section{Sensitivity analysis}

In the cohorts matched using traditional, non-time dependent propensity score matching, AAM was associated with worse outcomes in both rhythm cohorts (supplementary table A). The adjusted risk ratios were similar to the results of multivariable regression analysis in the original cohorts.

\section{Discussion}

In this large cohort study with time dependent propensity score sequential matching of adults with out-of-hospital cardiac arrest, the association of AAM with favourable outcomes depended on the first documented electrocardiographic rhythm (shockable or non-shockable). AAM did not show any advantage for shockable rhythm, whereas for non-shockable rhythm AAM was associated with higher one month survival.

\section{Relation to previous studies}

In previous observational studies, pre-hospital AAM by EMS personnel was associated with worse outcomes. $^{28} 29$ A previous study using traditional propensity score matching in the Japanese nationwide registry reported that AAM was associated with worse outcomes than bag-valve-mask ventilation. ${ }^{5}$ However, some of these previous observational studies may have had biased results favouring no AAM because patients who received AAM were likely to have needed AAM because they were more severely ill before arrest, a status that is potentially associated with less favourable outcomes. In addition, patients who received AAM during cardiopulmonary resuscitation would never have achieved return of spontaneous circulation before receiving AAM, a phenomenon now known as "resuscitation time bias" that would favour better outcomes in the no AAM group. ${ }^{8}$ Other recent 


\begin{tabular}{|c|c|c|c|c|c|c|}
\hline \multirow[b]{2}{*}{ Characteristics } & \multicolumn{3}{|l|}{ Shockable rhythm } & \multicolumn{3}{|l|}{ Non-shockable rhythm } \\
\hline & No AAM $(n=8057)$ & AAM $(n=8057)$ & Standardised difference & No AAM $(n=118021)$ & AAM $(n=118021)$ & Standardised difference \\
\hline Year: & & & 0.01 & & & 0.009 \\
\hline 2014 & 2731 (33.9) & $2776(34.5)$ & & $39342(33.3)$ & $39688(33.6)$ & \\
\hline 2015 & $2627(32.6)$ & $2604(32.3)$ & & $39214(33.2)$ & $39351(33.3)$ & \\
\hline 2016 & $2699(33.5)$ & $2677(33.2)$ & & $39465(33.4)$ & $38982(33.0)$ & \\
\hline Season: & & & 0.05 & & & 0.007 \\
\hline Spring & $1886(23.4)$ & $1914(23.8)$ & & $29051(24.6)$ & $29275(24.8)$ & \\
\hline Summer & $1678(20.8)$ & $1812(22.5)$ & & $21661(18.4)$ & $21808(18.5)$ & \\
\hline Autumn & $1867(23.2)$ & $1850(23.0)$ & & $26629(22.6)$ & $26636(22.6)$ & \\
\hline Winter & $2626(32.6)$ & $2481(30.8)$ & & $40680(34.5)$ & $40302(34.1)$ & \\
\hline Day: & & & 0.02 & & & 0.004 \\
\hline Weekday (Monday-Friday) & $5698(70.7)$ & $5634(69.9)$ & & $83106(70.4)$ & $83323(70.6)$ & \\
\hline Weekend (Saturday-Sunday) & $2359(29.3)$ & $2423(30.1)$ & & $34915(29.6)$ & $34698(29.4)$ & \\
\hline Time: & & & 0.03 & & & 0.004 \\
\hline Daytime (9:00-16:59) & $3123(38.8)$ & $3230(40.1)$ & & 41201 (34.9) & $41409(35.1)$ & \\
\hline Night time (17:00-8:59) & $4934(61.2)$ & 4827 (59.9) & & $76820(65.1)$ & $76612(64.9)$ & \\
\hline $\begin{array}{l}\text { Prefecture preference categories } \\
\text { for performing supraglottic airway } \\
\text { placement: }\end{array}$ & & & 0.08 & & & 0.01 \\
\hline$<15 \%$ & $240(3.0)$ & $255(3.2)$ & & $7456(6.3)$ & $7634(6.5)$ & \\
\hline $15 \%$ to $<30 \%$ & $2241(27.8)$ & $2119(26.3)$ & & $28978(24.6)$ & $29299(24.8)$ & \\
\hline $30 \%$ to $<45 \%$ & $1397(17.3)$ & $1225(15.2)$ & & $27360(23.2)$ & $26802(22.7)$ & \\
\hline$\geq 45 \%$ & $4179(51.9)$ & $4458(55.3)$ & & $54227(45.9)$ & $54286(46.0)$ & \\
\hline $\begin{array}{l}\text { Prefecture preference categories for } \\
\text { performing endotracheal intubation: }\end{array}$ & & & 0.06 & & & 0.02 \\
\hline$<1 \%$ & $5617(69.7)$ & $5476(68.0)$ & & $33101(28.0)$ & $33557(28.4)$ & \\
\hline $5 \%$ to $<10 \%$ & $610(7.6)$ & $551(6.8)$ & & $46552(39.4)$ & $45326(38.4)$ & \\
\hline $10 \%$ to $<15 \%$ & $977(12.1)$ & 1099 (13.6) & & $6008(5.1)$ & $6054(5.1)$ & \\
\hline$\geq 15 \%$ & $853(10.6)$ & $931(11.6)$ & & $32360(27.4)$ & $33084(28.0)$ & \\
\hline Median (IQR) age, years & $70(60-79)$ & $69(59-79)$ & 0.06 & $80(70-87)$ & $80(70-87)$ & 0.001 \\
\hline Sex: & & & 0.01 & & & 0.003 \\
\hline Female & $1723(21.4)$ & 1761 (21.9) & & $51105(43.3)$ & $51285(43.5)$ & \\
\hline Male & $6334(78.6)$ & $6296(78.1)$ & & $66916(56.7)$ & $66736(56.5)$ & \\
\hline Cause of cardiac arrest: & & & 0.05 & & & 0.005 \\
\hline Medical & 7464 (92.6) & 7555 (93.8) & & $91742(77.7)$ & $91519(77.5)$ & \\
\hline Non-medical & $593(7.4)$ & $502(6.2)$ & & $26279(22.3)$ & $26502(22.5)$ & \\
\hline Witness status: & & & 0.08 & & & 0.006 \\
\hline No witness & $2841(35.3)$ & $2580(32.0)$ & & $76284(64.6)$ & $76242(64.6)$ & \\
\hline By family & $3412(42.3)$ & $3494(43.4)$ & & $26443(22.4)$ & $26495(22.4)$ & \\
\hline By friend & $361(4.5)$ & $401(5.0)$ & & $1388(1.2)$ & $1369(1.2)$ & \\
\hline By colleague & $379(4.7)$ & $456(5.7)$ & & $899(0.8)$ & $897(0.8)$ & \\
\hline By passer-by & $330(4.1)$ & $365(4.5)$ & & $1422(1.2)$ & $1359(1.2)$ & \\
\hline By others & 734 (9.1) & $761(9.4)$ & & $11585(9.8)$ & $11659(9.9)$ & \\
\hline Type of bystander CPR: & & & 0.08 & & & 0.007 \\
\hline No bystander CPR & $3842(47.7)$ & $3569(44.3)$ & & $54672(46.3)$ & $54846(46.5)$ & \\
\hline $\begin{array}{l}\text { Chest compression only CPR with } \\
\text { dispatcher instruction }\end{array}$ & $3004(37.3)$ & $3176(39.4)$ & & $46791(39.6)$ & $46508(39.4)$ & \\
\hline $\begin{array}{l}\text { Chest compression only CPR without } \\
\text { dispatcher instruction }\end{array}$ & $748(9.3)$ & $771(9.6)$ & & $9397(8.0)$ & $9367(7.9)$ & \\
\hline $\begin{array}{l}\text { Conventional CPR with dispatcher } \\
\text { instruction }\end{array}$ & $297(3.7)$ & $373(4.6)$ & & $4939(4.2)$ & $5058(4.3)$ & \\
\hline $\begin{array}{l}\text { Conventional CPR without dispatcher } \\
\text { instruction }\end{array}$ & $166(2.1)$ & $168(2.1)$ & & $2222(1.9)$ & $2242(1.9)$ & \\
\hline $\begin{array}{l}\text { Use of public access defibrillator by } \\
\text { bystander: }\end{array}$ & & & 0.04 & & & 0.001 \\
\hline Yes & $262(3.3)$ & $318(3.9)$ & & $995(0.8)$ & $1001(0.8)$ & \\
\hline No & 7795 (96.7) & 7739 (96.1) & & $117026(99.2)$ & $117020(99.2)$ & \\
\hline Pre-hospital involvement of physician: & & & 0.03 & & & 0.005 \\
\hline Yes & $377(4.7)$ & $432(5.4)$ & & $2812(2.4)$ & $2896(2.5)$ & \\
\hline No & $7680(95.3)$ & $7625(94.6)$ & & $115209(97.6)$ & $115125(97.5)$ & \\
\hline $\begin{array}{l}\text { Median (IQR) time from call to EMS } \\
\text { CPR, minutes }\end{array}$ & $9(7-11)$ & $9(7-11)$ & 0.06 & $9(7-11)$ & $9(7-11)$ & 0.008 \\
\hline Defibrillation by EMS before matching: & & & 0.19 & & & 0.02 \\
\hline Yes & $7022(87.2)$ & $7480(92.8)$ & & $2020(1.7)$ & $1715(1.5)$ & \\
\hline $\begin{array}{l}\text { Median (IQR) time from EMS CPR } \\
\text { to defibrillation, minutes }\end{array}$ & $1(1-2)$ & $1(1-2)$ & & $5(2-8)$ & $5(3-8)$ & \\
\hline No & $1035(12.8)$ & $577(7.2)$ & & $116001(98.3)$ & $116306(98.5)$ & \\
\hline
\end{tabular}




\begin{tabular}{|c|c|c|c|c|c|c|}
\hline \multirow[b]{2}{*}{ Characteristics } & \multicolumn{3}{|l|}{ Shockable rhythm } & \multicolumn{3}{|l|}{ Non-shockable rhythm } \\
\hline & No AAM $(n=8057)$ & AAM $(n=8057)$ & Standardised difference & No AAM $(n=118021)$ & AAM $(n=118021)$ & Standardised difference \\
\hline $\begin{array}{l}\text { Administration of adrenaline by EMS } \\
\text { before matching: }\end{array}$ & & & 0.03 & & & 0.03 \\
\hline Yes & $687(8.5)$ & $766(9.5)$ & & $4072(3.5)$ & $4742(4.0)$ & \\
\hline $\begin{array}{l}\text { Median (IQR) time from EMS CPR } \\
\text { to adrenaline administration, minutes }\end{array}$ & $9(7-12)$ & $9(7-13)$ & & $9(7-13)$ & $9(7-13)$ & \\
\hline No & $7370(91.5)$ & $7291(90.5)$ & & $113949(96.5)$ & $113279(96.0)$ & \\
\hline Time from EMS CPR to matching: & & & $<0.001$ & & & $<0.001$ \\
\hline$\leq 4$ minutes & $1446(17.9)$ & $1446(17.9)$ & & $23188(19.6)$ & $23188(19.6)$ & \\
\hline 5 to $<10$ minutes & $3520(43.7)$ & $3520(43.7)$ & & $49205(41.7)$ & $49205(41.7)$ & \\
\hline 10 to $<15$ minutes & $1928(23.9)$ & $1928(23.9)$ & & $29007(24.6)$ & $29007(24.6)$ & \\
\hline 15 to $<20$ minutes & $753(9.3)$ & $753(9.3)$ & & $11298(9.6)$ & $11298(9.6)$ & \\
\hline 20 to $<25$ minutes & $254(3.2)$ & $254(3.2)$ & & $3563(3.0)$ & $3563(3.0)$ & \\
\hline 25 to $<30$ minutes & $111(1.4)$ & $111(1.4)$ & & $1166(1.0)$ & $1166(1.0)$ & \\
\hline$\geq 30$ minutes & $45(0.6)$ & $45(0.6)$ & & $594(0.5)$ & $594(0.5)$ & \\
\hline
\end{tabular}

$\mathrm{AAM}=$ advanced airway management; $C P R=$ cardiopulmonary resuscitation; EMS=emergency medical services; EMS CPR=cardiopulmonary resuscitation by emergency medical services personnel;

$I Q R=$ interquartile range.

cardiac arrest studies have used sequential matching with time dependent propensity scores to mitigate resuscitation time bias. ${ }^{101314}$

\section{Strengths and weaknesses of study}

Our study has several strengths. Firstly, its population based design using the nationwide registry enabled us to do the final analysis with complete data and no missing information. Secondly, the large sample size allowed us to estimate the associations between AAM and one month survival even when we divided our cohort by the first documented electrocardiographic rhythm. Thirdly, to overcome resuscitation time bias, we used time dependent propensity score sequential matching analysis. With this method, the groups were closely matched with respect to the timing of AAM. If we had not considered the timing of AAM, we would have had different results, as we showed in the multivariable regression analysis and in the sensitivity analysis using traditional propensity score matching (see supplementary material). We believe that this difference between the results is evidence of the presence of resuscitation time bias. Our approach is believed to have a lower risk of bias in its results compared with previous studies. ${ }^{56}$

Our study also has some limitations, however. Firstly, we did not have data about whether the first documented electrocardiographic rhythm changed to another rhythm during cardiopulmonary resuscitation.
Patients with non-shockable rhythm are thought to be likely to receive AAM more frequently and earlier than those with shockable rhythm, according to the algorithms of the international guidelines. ${ }^{20} 21$ Nevertheless, patients in both of our cohorts had similar proportions and timing of AAM. This might indicate that many patients whose first documented electrocardiographic rhythm is shockable develop a non-shockable rhythm during cardiopulmonary resuscitation and subsequently receive AAM according to the non-shockable algorithm. As post-defibrillation changes to non-shockable rhythms are common, and such patients have poorer outcomes than patients without rhythm changes, ${ }^{30} 31$ this would bias the results towards favouring no AAM among patients with a shockable first documented electrocardiographic rhythm.

Secondly, the number of AAM attempts per patient, especially for endotracheal intubation, and information on unsuccessful intubation were not available in our registry. Failed intubation is known to be associated with poor outcomes. ${ }^{32}$ If AAM was delayed, therefore, the patient could be matched in the no AAM group, and this could bias the results towards favouring the AAM group. The lack of information about proportion of successful intubation also decreases the generalisability of our study outside Japan. Thirdly, our database did not contain information on whether each patient was treated by a

\begin{tabular}{|c|c|c|c|c|}
\hline \multirow[b]{2}{*}{ Outcomes* } & \multicolumn{2}{|c|}{ No (\%) patients with outcome/total patients } & \multirow{2}{*}{$\begin{array}{l}\text { Unadjusted risk ratio } \\
(95 \% \mathrm{Cl})\end{array}$} & \multirow{2}{*}{$\begin{array}{l}\text { Adjusted risk ratiot } \\
(95 \% \mathrm{Cl})\end{array}$} \\
\hline & No AAM & AAM & & \\
\hline Survival & $1500 / 8057(18.6)$ & $1546 / 8057(19.2)$ & 1.09 (1.02 to 1.17$)$ & $1.00(0.93$ to 1.07$)$ \\
\hline Favourable functional survival & $865 / 8057(10.7)$ & $776 / 8057(9.6)$ & 0.97 (0.88 to 1.07$)$ & $0.87(0.79$ to 0.96$)$ \\
\hline \multicolumn{5}{|l|}{ Non-shockable rhythm } \\
\hline Favourable functional survival & $489 / 118021(0.4)$ & 499/118021 (0.4) & $1.10(0.96$ to 1.26$)$ & $1.11(0.97$ to 1.26$)$ \\
\hline
\end{tabular}




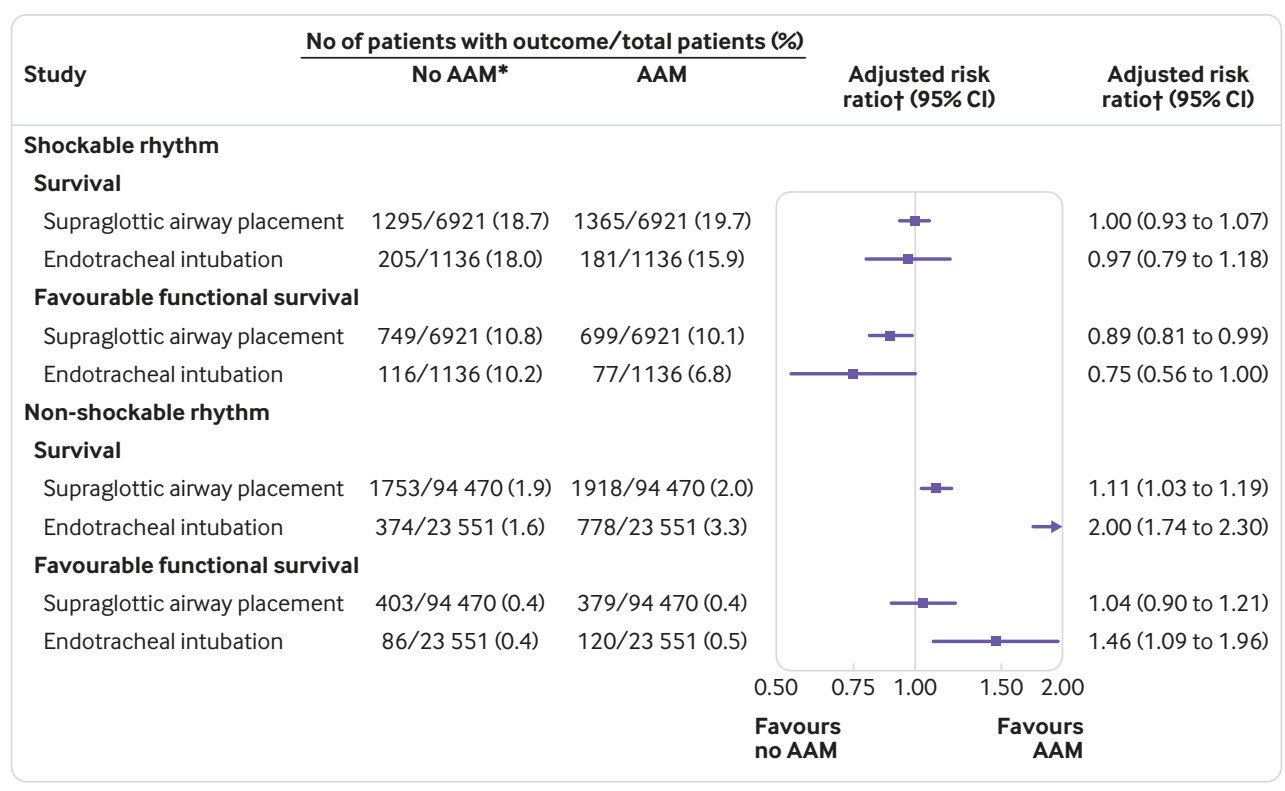

Fig 2 | Analysis stratified by type of advanced airway management (AAM) in matched cohort. * Matched control at same timing of supraglottic airway placement or endotracheal intubation. †Adjusted for prefecture preference categories for performing supraglottic airway placement and endotracheal intubation, year, season, day, time, age, sex, cause of cardiac arrest, witness category, basic life support by bystander with or without dispatcher instruction, use of public access defibrillator, pre-hospital involvement of physician, time from call to cardiopulmonary resuscitation by emergency medical services personnel, defibrillation before matching, and administration of adrenaline before matching

specially trained emergency life saving technician. As endotracheal intubation is performed only by these technicians and they are generally more experienced and trained than ordinary technicians, this could bias the results towards favouring AAM. Fourthly, we could not address potential confounding by indication in this study. For example, our database did not have any information about patients' comorbidities that could affect the decisions of EMS personnel. If patients with more significant comorbidities were less likely to receive interventions including AAM, this could bias the results towards favouring AAM. In contrast, if patients were more severely ill before arrest and more likely to have received AAM, this confounding could favour better outcomes in the no AAM group.

Meaning of study: possible explanations and implications for clinicians and policy makers In this study, we separated our original cohort by the first documented electrocardiographic rhythm because the international guidelines suggest different approaches in the algorithms for shockable and nonshockable rhythms. ${ }^{2021}$ Although the association in our sequential matching analysis was much weaker than the associations in the original cohort analysis and the traditional propensity score matching analysis, AAM was not associated with better one month survival and was still associated with a lower chance of favourable functional survival among patients with shockable rhythm. Among patients with non-shockable rhythm, in contrast, AAM was associated with better survival. Similarly, in a recent observational study of in-hospital cardiac arrest, a significant interaction affecting the primary outcome, survival to hospital discharge, was seen between patients with initial shockable and non-shockable rhythms. ${ }^{14}$ On the basis of our results, different airway management strategies for shockable and non-shockable rhythms should be more strongly emphasised; immediate defibrillation and continuous chest compression rather than ventilatory support are essential for patients with shockable rhythm, as recommended in the guidelines, ${ }^{20} 21$ whereas those with non-shockable rhythm could benefit from delivery of oxygen with protected airway ventilation.

\section{Unanswered questions and future research}

A recently published randomised controlled trial, in which all pre-hospital endotracheal intubation was done by experienced emergency physicians with a very low intubation failure proportion of $2 \%$, failed to show non-inferiority of bag-valve-mask ventilation relative to endotracheal intubation, but bag-valvemask ventilation was associated with complications such as regurgitation. ${ }^{7}$ Two recent randomised controlled trials compared pre-hospital supraglottic airway placement and endotracheal intubation during cardiopulmonary resuscitation. ${ }^{33} 34$ One showed that supraglottic airway placement was not significantly superior to endotracheal intubation, ${ }^{33}$ whereas the other reported that supraglottic airway placement was more effective in terms of patient outcomes than endotracheal intubation. ${ }^{34}$ In the second trial, however, the proportion of successful initial endotracheal intubation was low at 51\%. ${ }^{34}$ These results imply that the findings of future trials conducted under circumstances that allow for 
higher proportions of successful intubation might be different. In addition, no studies have investigated the effectiveness of supraglottic airway placement compared with bag-valve-mask ventilation. Given the findings of these recent trials, future research needs to compare supraglottic airway placement and bag-valvemask ventilation.

\section{Conclusions}

In this study of 310620 cases of out-of-hospital cardiac arrest in adults in a Japanese nationwide, population based out-of-hospital cardiac arrest registry, results differed according to the patient's first documented electrocardiographic rhythm. After time dependent propensity score sequential matching, AAM was not associated with survival in the shockable cohort but was associated with better survival in the nonshockable cohort. When we did not account for the timing of AAM and used multivariable regression or traditional propensity score matching, the associations suggested an unfavourable effect of AAM and were similar to the results of previous observational studies. Our findings suggest that different airway management strategies should be emphasised on the basis of initial electrocardiographic rhythm. Our results also imply that we should account for the timing of interventions in observational research.

\section{AUTHOR AFFILIATIONS}

Department of Anesthesiology, The Jikei University School of Medicine, Tokyo 105-8491, Japan

${ }^{2}$ Center for Critical Care Nephrology, Department of Critical Care Medicine, University of Pittsburgh, Pittsburgh, PA, USA

${ }^{3}$ Division of Biomedical Statistics, Department of Integrated Medicine, Graduate School of Medicine, Osaka University, Osaka, Japan

${ }^{4}$ Department of Emergency Medicine, Okinawa Chubu Hospital, Okinawa, Japan

${ }^{5}$ Department of Emergency Medicine, University of Pittsburgh, Pittsburgh, PA, USA

${ }^{6}$ Department of Food Science, Otsuma Women's University, Tokyo, Japan

${ }^{7}$ Department of Critical Care Nursing, Kyoto University Graduate School of Human Health Science, Kyoto, Japan

${ }^{8}$ Kyoto University Health Service, Kyoto, Japan

${ }^{9}$ Department of Emergency Medicine, Kyoto Prefectural University of Medicine, Kyoto, Japan

${ }^{10}$ Division of Environmental Medicine and Population Sciences, Department of Social and Environmental Medicine, Graduate School of Medicine, Osaka University, Osaka, Japan

We acknowledge all the EMS personnel and involved physicians in Japan and the staff of the Fire and Disaster Management Agency and the Institute for Fire Safety and Disaster Preparedness of Japan for their cooperation in establishing and maintaining the Utstein database. Contributors: II designed the study, analysed the data, and wrote the first draft of the manuscript. SK, KG, and TKitamura reviewed all statistical analyses and critically revised the manuscript. $\mathrm{MO}, \mathrm{KK}, \mathrm{CN}$, TKiguchi, TM, TKawamura, TI, and CWC supervised the analysis of the data and critically revised the manuscript. All the authors read and approved the final manuscript. The corresponding author attests that all listed authors meet authorship criteria and that no others meeting the criteria have been omitted. $J$ is the guarantor

Funding: This study was an investigator initiated study supported by the Clinical Investigator's Research Project at Osaka University Graduate School of Medicine and scientific research grants from the Ministry of Education, Culture, Sports, Science and Technology of Japan (16K09034 and 15H05006). This paper presents independent research supported by the funds.
Competing interests: All authors have completed the ICMJE uniform disclosure form at www.icmje.org/coi disclosure.pdf (available on request from the corresponding author) and declare: no support from any organisation for the submitted work other than that described above; no financial relationships with any organisations that might have an interest in the submitted work in the previous three years; and no other relationships or activities that could appear to have influenced the submitted work.

Ethical approval: The medical institutional review board of Osaka University Graduate School of Medicine approved this study and waived the need for informed consent because only de-identified data were used.

Data sharing: No additional data available.

Transparency: The lead author (JI) affirms that the manuscript is an honest, accurate, and transparent account of the study being reported; that no important aspects of the study have been omitted and that any discrepancies from the study as planned (and, if relevant, registered) have been explained.

This is an Open Access article distributed in accordance with the Creative Commons Attribution Non Commercial (CC BY-NC 4.0) license, which permits others to distribute, remix, adapt, build upon this work non-commercially, and license their derivative works on different terms, provided the original work is properly cited and the use is noncommercial. See: http://creativecommons.org/licenses/by-nc/4.0/.

1 Myat A, Song KJ, Rea T. Out-of-hospital cardiac arrest: current concepts. Lancet 2018;391:970-9. doi:10.1016/S01406736(18)30472-0

2 Iwami T, Kitamura T, Kiyohara K, Kawamura T. Dissemination of Chest Compression-Only Cardiopulmonary Resuscitation and Survival After Out-of-Hospital Cardiac Arrest. Circulation 2015;132:415-22. doi:10.1161/CIRCULATIONAHA.114.014905

3 Kitamura T, Kiyohara K, Sakai T, et al. Public-Access Defibrillation and Out-of-Hospital Cardiac Arrest in Japan. N Engl J Med 2016:375:1649-59. doi:10.1056/NEIMsa1600011

4 Ong MEH, Perkins GD, Cariou A. Out-of-hospital cardiac arrest: prehospital management. Lancet 2018;391:980-8. doi:10.1016/ S0140-6736(18)30316-7

5 Hasegawa K, Hiraide A, Chang Y, Brown DF. Association of prehospita advanced airway management with neurologic outcome and survival in patients with out-of-hospital cardiac arrest. JAMA 2013;309:25766. doi:10.1001/jama.2012.187612

6 McMullan J, Gerecht R, Bonomo J, et al, CARES Surveillance Group. Airway management and out-of-hospital cardiac arrest outcome in the CARES registry. Resuscitation 2014;85:617-22. doi:10.1016/j. resuscitation.2014.02.007

7 Jabre P, Penaloza A, Pinero D, et al. Effect of Bag-Mask Ventilation vs Endotracheal Intubation During Cardiopulmonary Resuscitation on Neurological Outcome After Out-of-Hospital Cardiorespiratory Arrest: A Randomized Clinical Trial. JAMA 2018;319:779-87. doi:10.1001/ jama.2018.0156

8 Andersen LW, Grossestreuer AV, Donnino MW. "Resuscitation time bias"-A unique challenge for observational cardiac arrest research. Resuscitation 2018;125:79-82. doi:10.1016/j. resuscitation.2018.02.006

9 Hagihara A, Hasegawa M, Abe T, Nagata T, Wakata Y, Miyazaki S. Prehospital epinephrine use and survival among patients with outof-hospital cardiac arrest. JAMA 2012;307:1161-8. doi:10.1001/ jama.2012.294

10 Nakahara S, Tomio J, Takahashi H, et al. Evaluation of pre-hospital administration of adrenaline (epinephrine) by emergency medical services for patients with out of hospital cardiac arrest in Japan: controlled propensity matched retrospective cohort study. BM/ 2013;347:f6829. doi:10.1136/bmj.f6829

11 Perkins GD, ji C, Deakin CD et al, PARAMEDIC2 Collaborators. A Randomized Trial of Epinephrine in Out-of-Hospital Cardiac Arrest. N Engl / Med 2018;379:711-21. doi:10.1056/NEJMoa1806842

12 Andersen LW, Granfeldt A. Epinephrine in cardiac arrest - insights from observational studies. Resuscitation 2018;131:e1. doi:10.1016/j.resuscitation.2018.07.028

13 Andersen LW, Raymond TT, Berg RA, et al, American Heart Association's Get With The Guidelines-Resuscitation Investigators. Association Between Tracheal Intubation During Pediatric In-Hospital Cardiac Arrest and Survival. JAMA 2016:316:1786-97. doi:10.1001/ jama.2016.14486

14 Andersen LW, Granfeldt A, Callaway CW, et al, American Heart Association's Get With The Guidelines-Resuscitation Investigators. Association Between Tracheal Intubation During Adult In-Hospital Cardiac Arrest and Survival. JAMA 2017;317:494-506. doi:10.1001/ jama.2016.20165

15 Jacobs I, Nadkarni V, Bahr J, et al, International Liason Committee on Resusitation. Cardiac arrest and cardiopulmonary resuscitation 
outcome reports: update and simplification of the Utstein templates for resuscitation registries. A statement for healthcare professionals from a task force of the international liaison committee on resuscitation (American Heart Association, European Resuscitation Council, Australian Resuscitation Council, New Zealand Resuscitation Council, Heart and Stroke Foundation of Canada, InterAmerican Heart Foundation, Resuscitation Council of Southern Africa). Resuscitation 2004;63:233-49. doi:10.1016/j. resuscitation.2004.09.008

16 Kitamura T, Iwami T, Kawamura T, Nagao K, Tanaka H, Hiraide A, Implementation Working Group for the All-Japan Utstein Registry of the Fire and Disaster Management Agency. Nationwide public access defibrillation in Japan. N Engl J Med 2010;362:994-1004 doi:10.1056/NEJMoa0906644

17 Japan Resuscitation Council. 2010 Japanese guidelines for emergency care and cardiopulmonary resuscitation. Health Shuppansha, 2011.

18 Nolan JP, Hazinski MF, Billi JE, et al. Part 1: Executive summary: 2010 International Consensus on Cardiopulmonary Resuscitation and Emergency Cardiovascular Care Science With Treatment Recommendations. Resuscitation 2010;81(Suppl 1):e1-25. doi:10.1016/j.resuscitation.2010.08.002

19 Perkins GD, Jacobs IG, Nadkarni VM, et al, Utstein Collaborators. Cardiac Arrest and Cardiopulmonary Resuscitation Outcome Reports: Update of the Utstein Resuscitation Registry Templates for Out-ofHospital Cardiac Arrest: A Statement for Healthcare Professionals From a Task Force of the International Liaison Committee on Resuscitation (American Heart Association, European Resuscitation Council, Australian and New Zealand Council on Resuscitation, Heart and Stroke Foundation of Canada, InterAmerican Heart Foundation, Resuscitation Council of Southern Africa, Resuscitation Council of Asia); and the American Heart Association Emergency Cardiovascular Care Committee and the Council on Cardiopulmonary, Critical Care, Perioperative and Resuscitation. Resuscitation 2015;96:328-40. doi:10.1016/j.resuscitation.2014.11.002

20 Soar J, Nolan JP, Böttiger BW, et al, Adult advanced life support section Collaborators. European Resuscitation Council Guidelines for Resuscitation 2015: Section 3. Adult advanced life support. Resuscitation 2015;95:100-47. doi:10.1016/j. resuscitation.2015.07.016

21 Link MS, Berkow LC, Kudenchuk PJ, et al. Part 7: Adult Advanced Cardiovascular Life Support: 2015 American Heart Association Guidelines Update for Cardiopulmonary Resuscitation and Emergency Cardiovascular Care. Circulation 2015;132(Suppl 2):S444-64. doi:10.1161/CIR.0000000000000261

22 Lu B. Propensity score matching with time-dependent covariates. Biometrics 2005;61:721-8. doi:10.1111/j.15410420.2005.00356.x
23 Beyersmann J, Schumacher M. Time-dependent covariates in the proportional subdistribution hazards model for competing risks. Biostatistics 2008:9:765-76. doi:10.1093/biostatistics/kxn009

24 Kalbfleisch JD, Prentice RL. The statistical analysis of failure time data. John Wiley \& Sons, 2011.

25 Li YFP, Propert KJ, Rosenbaum PR. Balanced risk set matching. J Am Stat Assoc 2001;96:870-82 doi:10.1198/016214501753208573.

26 Stuart EA. Matching methods for causal inference: A review and a look forward. Stat Sci 2010;25:1-21. doi:10.1214/09-STS313

27 Austin PC. An Introduction to Propensity Score Methods for Reducing the Effects of Confounding in Observational Studies. Multivariate Behav Res 2011:46:399-424. doi:10.1080/0027317 1.2011 .568786

28 Fouche PF, Simpson PM, Bendall J, Thomas RE, Cone DC, Doi SA. Airways in out-of-hospital cardiac arrest: systematic review and metaanalysis. Prehosp Emerg Care 2014;18:244-56. doi:10.3109/1090 3127.2013.831509

29 Jeong S, Ahn KO, Shin SD. The role of prehospital advanced airway management on outcomes for out-of-hospital cardiac arrest patients: a meta-analysis. Am J Emerg Med 2016;34:2101-6. doi:10.1016/j. ajem.2016.07.025

30 Niemann JT, Stratton SI, Cruz B, Lewis RJ. Outcome of out-ofhospital postcountershock asystole and pulseless electrical activity versus primary asystole and pulseless electrical activity. Crit Care Med 2001;29:2366-70. doi:10.1097/00003246-20011200000020

31 Mehta C, Brady W. Pulseless electrical activity in cardiac arrest: electrocardiographic presentations and management considerations based on the electrocardiogram. Am J Emerg Med 2012;30:236-9. doi:10.1016/j.ajem.2010.08.017

$32 \mathrm{Kim} \mathrm{J,} \mathrm{Kim} \mathrm{K,} \mathrm{Kim} \mathrm{T,} \mathrm{et} \mathrm{al.} \mathrm{The} \mathrm{clinical} \mathrm{significance} \mathrm{of} \mathrm{a} \mathrm{failed} \mathrm{initial}$ intubation attempt during emergency department resuscitation of out-of-hospital cardiac arrest patients. Resuscitation 2014;85:623-7. doi:10.1016/j.resuscitation.2014.01.017

33 Benger JR, Kirby K, Black S, et al. Effect of a Strategy of a Supraglottic Airway Device vs Tracheal Intubation During Out-ofHospital Cardiac Arrest on Functional Outcome: The AIRWAYS-2 Randomized Clinical Trial. JAMA 2018;320:779-91. doi:10.1001/ jama.2018.11597

34 Wang HE, Schmicker RH, Daya MR, et al. Effect of a Strategy of Initial Laryngeal Tube Insertion vs Endotracheal Intubation on 72-Hour Survival in Adults With Out-of-Hospital Cardiac Arrest: A Randomized Clinical Trial. JAMA 2018;320:769-78. doi:10.1001/ jama.2018.7044

35 Andersen LW, Granfeldt A. Pragmatic Airway Management in Outof-Hospital Cardiac Arrest. JAMA 2018;320:761-3. doi:10.1001/ jama.2018.10824 